\title{
An Enhanced Spanning Tree Topology for Wireless Sensor Networks
}

\author{
Smita A. Attarde \\ Lokmanya Tilak College of Engg., \\ Navi Mumbai, India
}

\author{
Lata L. Ragha \\ Ramrao Adik Institute of Technology, \\ Nerul, Navi Mumbai, India
}

\author{
Shital K. Dhamal \\ Lokmanya Tilak College of Engg., \\ Navi Mumbai, India
}

\begin{abstract}
Most of the parallel and distributed applications are subject to frequent disruptions due to resource contention and failure. Such disruptions are inherently unpredictable and, therefore, robustness is a desirable property for the distributed operating environment. An approach to robustness is considered for applications that operate on a spanning tree overlay network. For such applications, a robust spanning tree is designed by considering a weighted combination of hop count and path weight. The robust spanning tree topology shows good performance when applied to wireless sensor network. For wireless sensor networks, the expected data loss and power consumption metrics are very important. When both of these metrics are considered, the proposed robust tree shows average results as compared with the existing spanning tree topologies. When a node fails, the expected data loss is also average when compared with the existing trees like Dijkstra's, fewest hop and Prim's minimum spanning tree topologies.
\end{abstract}

The full text of the article is not available in the cache. Kindly refer the IJCA digital library at www.ijcaonline.org for the complete article. In case, you face problems while downloading the full-text, please send a mail to editor at editor@ijcaonline.org 